\title{
AVALIAÇÃO DE CINCO SELEÇÕES DE LARANJEIRA 'VALÊNCIA' SOBRE DOIS PORTA-ENXERTOS ${ }^{1}$
}

\author{
FABIO TOMASETTO², EDUARDO SANCHES STUCHI ${ }^{3}$, ANTONIO BALDO GERALDO MARTINS ${ }^{4}$
}

RESUMO-O presente trabalho teve por objetivo avaliar cinco seleções de laranjeira 'Valência', quanto ao potencial industrial e de consumo in natura com relação às variedades 'Natal' e 'Valência', enxertadas sobre o citrumeleiro 'Swingle' (experimento I) e a tangerineira 'Sunki' (experimento II). Os experimentos foram conduzidos na Estação Experimental de Citricultura de Bebedouro (E.E.C.B.)-SP. A implantação dos experimentos foi em fevereiro de 2001, sob espaçamentos de 7,0 m entre linhas e 5,0 m entre plantas (285 plantas/ha) e 7,0 m entre linhas e 3,0 m entre plantas (476 plantas/ha), respectivamente, para os experimentos I e II. O delineamento experimental foi em blocos ao acaso, duas plantas por parcela, quatro repetições e 7 tratamentos, constituídos pelas seguintes variedades-copa: 1) 'Natal'; 2) 'Valência'; 3) 'Valência-Don João’; 4) ‘Valência-late-Burjasot’ IVIA 35-2; 5) ‘Valência-Rohde-Red’ SRA-36; 6) ‘Valência-Temprana’ IVIA25; 7) ‘Valência Camphell’. A seleção 'Valência late’ é a que se destaca por ser a que mais se assemelhou às variedades tradicionais Valência e Natal.

Termos para indexação: índice tecnológico, laranja, qualidade.

\section{EVALUATION OF FIVE VALENCIA SWEET ORANGES SELECTION ON TWO ROOTSTOCKS}

\begin{abstract}
The aim of the current work was to compare five Valencia sweet oranges selection with industrial potential and in nature consumption with the varieties 'Natal'and 'Valencia' on two rootstocks: 'Swingle' (experiment I) and 'Sunki’ (experiment II) when it comes as yield and with technological index. Two experiments were set up on February 2001, in Bebedouro city, São Paulo state, Brazil. The spacements were $7.0 \mathrm{~m}$ between rows with $5.0 \mathrm{~m}$ between trees and $7.0 \mathrm{~m}$ between rows with $3.0 \mathrm{~m}$ between trees, respectively for experiment I and experiment II. The experimental design used was randomized blocks, with four replications and two trees per plot. The crown varieties tested were 1) 'Natal', 2) 'Valência', 3) 'Valência Don João', 4) 'Valência late Burjasot’ IVIA 35-2, 5) ‘Valência Rohde Red’ SRA-36, 6) ‘Valência Temprana’ IVIA-25, 7) 'Valência Campbell'. The varieties of Valência studied proved some similarity for yield and technological index. The 'Valência Late' can be highlighted because it was the one that resembled to the traditional Valência and Natal varieties.
\end{abstract}

Index terms: technological index, orange, quality.

\section{INTRODUÇÃO}

O Brasil é o maior produtor de laranja do mundo, produzindo em 2007, aproximadamente, 447 milhões de caixas (Agrianual, 2008); além disso, lidera as exportações de suco concentrado, exportando, na safra de 2006/2007, 1.393.864 toneladas (Abecitrus, 2008).

O Estado de São Paulo é o maior produtor do Brasil, sendo responsável por $80 \%$ da produção nacional e $85 \%$ do suco concentrado comercializado no mercado internacional (Neves, 1997). Tem em seu território 211 milhões de pés de laranja, dos quais 180 milhões estão em produção (Agrianual, 2007). Atualmente, 70 a $80 \%$ da produção paulista destinase à industrialização e de 20 a $30 \%$ são comercializados in natura. Do total do suco produzido, 98\% é comercializado no mercado internacional, com a maior parte sendo engarrafada pelas empresas europeias e norte-americanas (Boteon \& Neves,2005).

Neves et al. (2003) relatam que a laranja possui a quinta maior renda bruta das principais culturas do Estado de São Paulo por unidade de

\footnotetext{
${ }^{1}$ (Trabalho 133-08). Recebido em: 27-05-2008. Aceito para publicação em: 13-01-2009. Apoio FAPESP (Proc.06/54640-7). ${ }^{2}$ Mestrando, Faculdade de Ciências Agrárias e Veterinárias - UNESP, Jaboticabal-SP. fabiotomasetto@yahoo.com.br ${ }^{3}$ Pesquisador da Embrapa Mandioca e Fruticultura, trabalhando na Estação Experimental de Citricultura de Bebedouro, Caixa Postal 74, 14700-000, Bebedouro-SP. stuchi@cnpmf.embrapa.br

${ }^{4}$ Prof Dr Assistente Departamento de Produção Vegetal , Faculdade de Ciências Agrárias e Veterinárias - UNESP, JaboticabalSP. baldo@fcav.unesp.br
} 
área. E que a inexistência da citricultura em território paulista deixaria de movimentar na economia brasileira cerca de 4 bilhões de dólares anuais.

Embora existam pesquisas em variedadescopa e portas-enxerto no País, a quantidade de opções para o produtor é ainda pequena, especialmente pela presença de muitas doenças (Domingues, 1998).

A ocorrência de problemas fitossanitários graves, como a clorose variegada dos citros (cvc), declínio, gomose, leprose, mancha-preta, alternaria e a morte súbita dos citros, vem acarretando muitos prejuízos ao setor citrícola. Diante dessa situação, demonstra-se a grande importância da obtenção de variedades e/ou combinações copa/porta-enxerto que apresentem tolerância a pragas e doenças, permitindo o suprimento da demanda de frutas pelo mercado.

Neste contexto, este trabalho avaliou cinco seleções da variedade Valência, quanto à produção e índice tecnológico, tendo como parâmetro as variedades tradicionais, Valência e Natal, como opção à citricultura nacional.

\section{MATERIAL E MÉTODOS}

Os experimentos foram conduzidos na Estação Experimental de Citricultura de Bebedouro (E.E.C.B.), município de Bebedouro-SP. O clima da região é subtropical, com inverno moderado e seco, verão quente e chuvoso, com temperatura média de $23,5^{\circ} \mathrm{C}$; a precipitação anual média é de $1.522 \mathrm{~mm}$. Os experimentos foram instalados em fevereiro de 2001, nos espaçamentos de 7,0 m entre linhas e 5,0 m entre plantas (285 plantas/ha) e 7,0 m entre linhas e 3,0m entre plantas (476 plantas/ha), respectivamente, para os experimentos I (variedades-copa enxertadas em 'Swingle') e II (variedades copas enxertadas em 'Sunki'). O delineamento experimental, para ambos, foi em blocos ao acaso, duas plantas por parcela, quatro repetições e 7 tratamentos, constituídos pelas seguintes variedades-copa: 1) 'Natal', 2) 'Valência', 3) 'Valência-Don João', 4) 'Valência-late-Burjasot' IVIA 35-2, 5) 'Valência-Rohde-Red' SRA-36, 6) 'Valência-Temprana' IVIA-25, 7) 'Valência-Campbell'.

A avaliação de produção foi realizada no ano de 2006. A colheita dos frutos e a pesagem foi efetuada por parcelas, calculando-se posteriormente a produtividade média, em kg. planta $^{-1}$. Os resultados obtidos foram submetidos à análise de variância. As médias obtidas do experimento foram comparadas pelo teste de Tukey, ao nível de 5\% de probabilidade. A qualidade dos frutos foi avaliada mensalmente, de julho a dezembro de 2006. Em cada avaliação, coletaram-se amostras de cinco frutos por parcela.
Com estas amostras, determinaram-se as seguintes características de qualidade dos frutos: massa, diâmetro e altura; e do suco: sólidos solúveis totais (SST), determinados com refratômetro de leitura direta e expressos em Brix; acidez por titulação com hidróxido de sódio 0,3125 N, expressa em g de ácido cítrico/100 mL; ratio, que é a razão aritmética entre os sólidos solúveis totais e a acidez, rendimento em suco (RS) expresso em porcentagem $(\mathrm{m} / \mathrm{m})$ e o índice tecnológico (IT), expresso em kg de sólidos solúveis totais por caixa $\left(\mathrm{kg} \mathrm{SST}\right.$. caixa ${ }^{-1}$ e calculado pela expressão: IT = [RS x SST x 40,8] x 10.000-1, sendo que o valor de $40,8 \mathrm{~kg}$ corresponde à da caixa-padrão industrial de citros. Os resultados obtidos foram submetidos à análise de variância, e as médias foram comparadas pelo teste de Tukey.

Foram efetuados os ajustes de equações polinomiais, para as variáveis SS, acidez e ratio, em função do número de dias acumulados após o início da primeira coleta de frutos para análise. Definiramse o valor zero para a primeira data de análise (11Julho) e, correspondentemente, os seguintes valores para as demais coletas: $37,56,87,121$ e153dias.

\section{RESULTADOS E DICUSSÃO}

Ao comparar as médias de produção, para variedades estudadas sobre citrumelo 'Swingle', não se observaram diferenças estatísticas. Já, no segundo experimento, das copas avaliadas, enxertadas em 'Sunki', nota-se que a 'Natal' se destaca, pois foi a que apresentou a maior produção, assemelhando-se aos tratamentos 'Valência-RohdeRed’e 'Valência'. Stuchi et al.(2002) relatam, para as mesma condições, uma produção kg/planta para a variedade Valência, aos 6 anos de idade e em espaçamento de 7 metros entre linhas e 4 metros entre plantas, maior sobre o porta-enxerto 'Swingle' e 'Sunki', respectivamente, de 59,27 e 69,63 kg planta-1. As médias de produção referente ao ano de 2006 encontram-se na Tabela 1

Comparando os valores médios para a análise de frutos, das seleções, no momento em que estas atingiram "ratio" 12, que é o mínimo indicado para a colheita e destino dos frutos à indústria (Viegas, 1991), observa-se que as médias, referentes à 'Valência-late' (Tabelas 2 e 3), destacam-se para ambos os experimentos, com exceção para índice tecnológico (IT) no experimento I, que se igualou estatisticamente à menor média obtida pela seleção 'Don João'. Para características físicas e químicas dos frutos analisadas acima, Stuchi et al. (2002) relatam valores muito similares para a variedade 
Valência sobre 'Swingle' e 'Sunki', como para o porta-enxerto ‘Swingle’: R.S. 51,3\% e I.T 2,65, já para o porta-enxerto 'Sunki': massa 192,5 g e 7,05 cm para diâmetro. Di Giorgi et al.(1990) descreveram valores superiores para R.S. e I.T, com seus respectivos valores de $56,1 \%$ e 2,74 para a variedade Valência e 56,2\% e 2,79 para Natal.

Ajuste de equações às variáveis químicas

As estimativas dos parâmetros das equações ajustadas referentes às variáveis adotadas no estudo ( ${ }^{\circ}$ Brix, acidez e ratio), para as 7 copas de laranja-doce, estão apresentadas nas Tabelas 4 e 5. As variáveis acidez e ratio apresentaram melhor ajuste ao modelo linear, enquanto, para a variável ${ }^{\circ}$ Brix, foi o modelo polinomial de segunda ordem. Resultados semelhantes foram observados por Mattos Junior et al. (1999), para 'Valência-late’, já

A) citrumeleiro Swingle
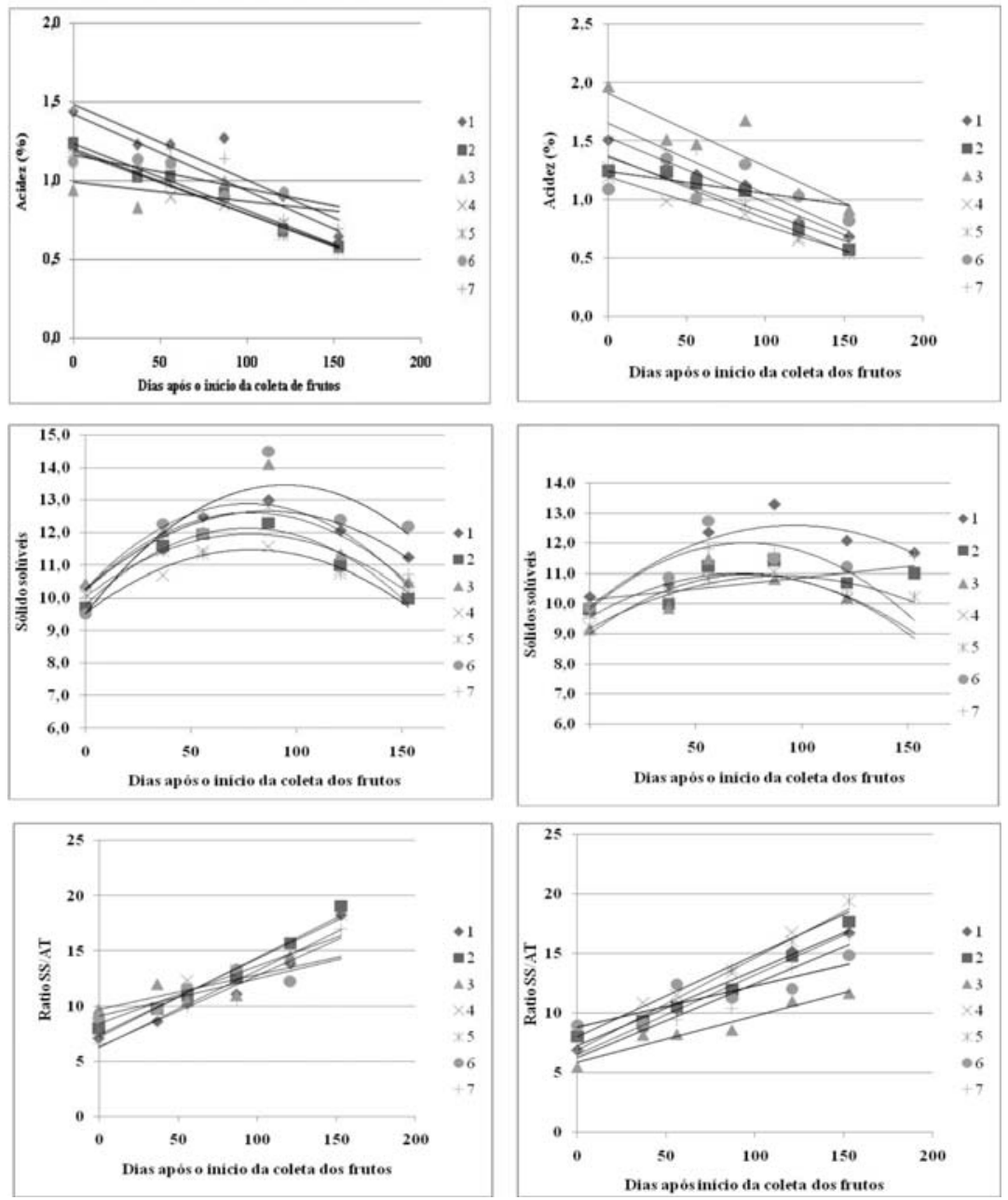

FIGURA1 - Evolução da acidez ('Swingle’-Ae ‘Sunki’- B), Sólidos solúveis ( ${ }^{\circ B r i x) ~(' S w i n g l e ’-~ C ~ e ~ ‘ S u n k i ’-~}$ D) e ratio ('Swingle'- E e 'Sunki'- F), para as variedades seleções ( 1- 'Natal', 2- 'Valência', 3'Valência-Don João’, 4- 'Valência-late-Burjasot’ IVIA 35-2, 5- 'Valência-Rohde-Red’ SRA-36, 6- 'Valência-Temprana’ IVIA-25, 7- 'Valência-Camphell’) estudadas. Bebedouro - SP. 
TABELA 1 - Médias observadas para produção para variedades estudadas sobre citrumelo 'Swingle’e tangerineira 'Sunki'. Bebedouro-SP, 2006.

\begin{tabular}{lcc}
\hline \multicolumn{2}{c}{ Tratamentos } & \multicolumn{2}{c}{ Produção } \\
& 'sginglanta & 'sunki' \\
\hline 1 Natal & $57,15 \mathrm{a}$ & $73,62 \mathrm{a}$ \\
2 Valência & $29,40 \mathrm{a}$ & $45,26 \mathrm{ab}$ \\
3 Don João & $26,03 \mathrm{a}$ & $40,56 \mathrm{~b}$ \\
4 Valência-late-Burjasot IVIA 35-2 & $45,27 \mathrm{a}$ & $17,17 \mathrm{~b}$ \\
5 Valência-Rohde-Red SRA-360 & $34,21 \mathrm{a}$ & $47,84 \mathrm{ab}$ \\
6 Valência-Temprana IVIA-25 & $44,75 \mathrm{a}$ & $24,75 \mathrm{~b}$ \\
7 Valência-Camphell & $36,99 \mathrm{a}$ & $38,90 \mathrm{~b}$ \\
\hline DMS & 36,10 & 32,08 \\
\hline CV (\%) & 32,97 & 33,38 \\
\hline
\end{tabular}

Médias seguidas pelas mesmas letras minúsculas na coluna não diferem, pelo teste de Tukey (5\%).

TABELA 2- Médias obtidas nas análises físicas e químicas para os frutos das variedades copa estudadas sobre citrumelo 'Swingle’.(Experimento I). Bebedouro-SP,

\begin{tabular}{|c|c|c|c|c|c|}
\hline \multirow[b]{2}{*}{ Tratame nto } & \multicolumn{5}{|c|}{ ANÁLISE DE FRUTOS } \\
\hline & $\begin{array}{c}\text { Massa } \\
\mathrm{g}\end{array}$ & $\begin{array}{c}\text { Diâmetro } \\
\mathrm{cm}\end{array}$ & $\begin{array}{c}\text { Altura } \\
\mathrm{cm}\end{array}$ & $\begin{array}{c}\text { Rend. Suco } \\
\%\end{array}$ & $\begin{array}{c}\mathrm{I} . \mathrm{T} . \\
\mathrm{Kg}(\mathrm{SS}) . \\
\mathrm{cx}^{-1}(40,8)\end{array}$ \\
\hline 1 Natal & $185,75 \mathrm{~cd}$ & $6,87 \mathrm{c}$ & $7,05 \mathrm{~cd}$ & $51,52 \mathrm{a}$ & $2,53 \mathrm{a}$ \\
\hline 2 Valência & $226,55 \mathrm{ab}$ & $7,30 \mathrm{ab}$ & $7,50 \mathrm{bc}$ & $53,41 \mathrm{a}$ & $2,33 \mathrm{ab}$ \\
\hline 3 Don João & $219,92 \mathrm{bc}$ & $7,60 \mathrm{ab}$ & $7,90 \mathrm{ab}$ & $35,86 \mathrm{c}$ & $1,51 \mathrm{c}$ \\
\hline 4 Valência-late-Burjasot IVIA 35 -2 & $252,97 \mathrm{a}$ & $8.12 \mathrm{a}$ & $8,25 \mathrm{a}$ & $50,76 \mathrm{ab}$ & $1,90 \mathrm{bc}$ \\
\hline 5 Valência-Rohde-Red SRA-360 & $178,35 \mathrm{~d}$ & $6,72 \mathrm{c}$ & $6,77 \mathrm{~d}$ & $55,44 \mathrm{a}$ & $2,30 \mathrm{ab}$ \\
\hline 6 Valência-Temprana IVIA-25 & $208,50 \mathrm{bcd}$ & $7,27 \mathrm{bc}$ & $7,32 \mathrm{bcd}$ & $43,69 \mathrm{~b}$ & $1,99 \mathrm{abc}$ \\
\hline 7 Valência-Camphell & $201,15 \mathrm{bcd}$ & $7,05 \mathrm{bc}$ & $7,30 \mathrm{~cd}$ & $52,48 \mathrm{a}$ & $2,23 \mathrm{ab}$ \\
\hline DMS & 39,06 & 0,63 & 0,57 & 6,26 & 0,63 \\
\hline CV $(\%)$ & 7,88 & 3,69 & 3,31 & 7,16 & 12,72 \\
\hline
\end{tabular}

Médias seguidas pelas mesmas letras minúsculas na coluna não diferem, pelo teste de Tukey (5\%). 
TABELA 3 - Médias obtidas nas análises físicas e químicas para os frutos das variedades-copa estudadas sobre tangerineira ‘Sunki’.(ExperimentoII) Bebedouro-SP, 2006.

\begin{tabular}{|c|c|c|c|c|c|}
\hline \multirow[b]{2}{*}{ Tratamento } & \multicolumn{5}{|c|}{ ANÁLISE DE FRUTOS } \\
\hline & $\begin{array}{c}\text { Massa } \\
\mathrm{g}\end{array}$ & $\begin{array}{c}\text { Diâmetro } \\
\mathrm{cm}\end{array}$ & $\begin{array}{l}\text { Altura } \\
\mathrm{cm}\end{array}$ & $\begin{array}{c}\text { Rend. Suco } \\
\%\end{array}$ & $\begin{array}{c}\text { I.T. } \\
\mathrm{Kg}(\mathrm{SS}) . \\
\mathrm{cx}^{-1}(40,8)\end{array}$ \\
\hline 1 Natal & $174,75 \mathrm{~b}$ & $6,72 b$ & $7,12 \mathrm{~b}$ & $50,81 \mathrm{a}$ & $2,50 \mathrm{a}$ \\
\hline 2 Valência & $194,75 \mathrm{~b}$ & $6,90 \mathrm{~b}$ & $7,40 \mathrm{~b}$ & $49,42 \mathrm{ab}$ & $2,21 \mathrm{ab}$ \\
\hline 3 Don João & $191,60 \mathrm{~b}$ & $7,00 \mathrm{ab}$ & $7,22 \mathrm{~b}$ & $44,12 \mathrm{bc}$ & $2,04 \mathrm{~b}$ \\
\hline 4 Valência-1Ate-Burjasot IVIA 35 -2 & $253,30 \mathrm{a}$ & $7,60 \mathrm{a}$ & 8,17 a & $48,53 \mathrm{abc}$ & $2,21 \mathrm{ab}$ \\
\hline 5 Valência-Rohde-Red SRA-360 & $198,75 \mathrm{~b}$ & $7,07 \mathrm{ab}$ & $7,22 \mathrm{~b}$ & $50,35 \mathrm{ab}$ & $2,20 \mathrm{a} \mathrm{b}$ \\
\hline 6 Valência-Temprana IVIA-25 & $202,80 \mathrm{~b}$ & $7,20 \mathrm{ab}$ & $7,22 \mathrm{~b}$ & $42,34 \mathrm{c}$ & $2,14 \mathrm{ab}$ \\
\hline 7 Valência-Camphell & $206,70 \mathrm{ab}$ & $7,17 \mathrm{ab}$ & $7,40 \mathrm{~b}$ & $49,29 \mathrm{ab}$ & $2,28 \mathrm{ab}$ \\
\hline DMS & 50,41 & 0,60 & 4,19 & 6,56 & 0,39 \\
\hline $\mathrm{CV}(\%)$ & 10,61 & 4,11 & 0,72 & 5,87 & 7,58 \\
\hline
\end{tabular}

Médias seguidas pelas mesmas letras minúsculas na coluna não diferem, pelo teste de Tukey (5\%).

TABELA 4 - Estimativas dos parâmetros das equações polinomiais ajustadas para exprimir a relação de ${ }^{\circ}$ Brix, acidez e ratio com o número de dias acumulados após o início da coleta de frutos, e correspondentes coeficientes de determinação, para as seleções e variedades de laranjas, enxertadas sobre citrumelo ‘Swingle’. (Experimento I ) Bebedouro-SP, 2006.

\begin{tabular}{|c|c|c|c|c|c|c|c|c|c|c|}
\hline \multirow{2}{*}{ Tratamento } & \multicolumn{4}{|c|}{${ }^{\circ}$ Brix $^{1}$} & \multicolumn{3}{|c|}{ Acidez $^{2}$} & \multicolumn{3}{|c|}{ Ratio $^{3}$} \\
\hline & $\mathrm{c}$ & $\mathrm{b}$ & $\mathrm{a}$ & $\mathrm{r}^{2}$ & $\mathrm{~b}$ & $\mathrm{a}$ & $\mathrm{r}^{2}$ & $\mathrm{~b}$ & $\mathrm{a}$ & $\mathrm{r}^{2}$ \\
\hline 1 & 9,9175 & 0,0558 & $-0,00029$ & $73,12^{*}$ & 1,4789 & $\begin{array}{l}- \\
0,004767\end{array}$ & $85,58^{*}$ & 6,3102 & 0,0693 & $94,13^{*}$ \\
\hline 2 & 9,6893 & 0,02777 & $-0,00013$ & $65,60 \mathrm{NS}$ & 1,2336 & $-0,00424$ & $97,37^{*}$ & 7,2609 & 0,07152 & $97,28^{*}$ \\
\hline 3 & 9,0189 & 0,05228 & $\begin{array}{l}- \\
0,000348\end{array}$ & $74,69^{*}$ & 0,9927 & $\begin{array}{l}- \\
0,001262\end{array}$ & $13,95 \mathrm{NS}$ & 9,7013 & 0,0314 & $61,76^{*}$ \\
\hline 4 & 9,5597 & 0,0409 & $\begin{array}{l}- \\
0,000290\end{array}$ & $90,31 *$ & 1,1835 & $-0,0039$ & $95,95^{*}$ & 8,4125 & 0,0523 & $93,63^{*}$ \\
\hline 5 & 9,1798 & 0,0386 & $\begin{array}{l}- \\
0,000215\end{array}$ & $75,42 *$ & 1,1986 & $-0,0041$ & $97,65^{*}$ & 7,4437 & 0,0688 & $99,54^{*}$ \\
\hline 6 & 9,8081 & 0,05965 & $\begin{array}{l}- \\
0,000405\end{array}$ & $69,38 \mathrm{NS}$ & 1,1650 & $\begin{array}{l}- \\
0,002142\end{array}$ & 72,54 NS & 9,1143 & 0,0341 & $74,28 *$ \\
\hline 7 & 9,5836 & 0,03492 & $\begin{array}{l}- \\
0,000163\end{array}$ & $53,04 *$ & 1,4167 & $-0,0048$ & $87,33^{*}$ & 6,3597 & 0,0642 & $95,13^{*}$ \\
\hline
\end{tabular}

$1{ }^{\circ}$ Brix $\mathrm{y}=\mathrm{c}+\mathrm{bx}+\mathrm{ax}^{2} ;{ }^{2}$ Acidez $(\%) \mathrm{y}=\mathrm{b}+\mathrm{ax} ;{ }^{3}$ Ratio $\mathrm{y}=\mathrm{b}+\mathrm{ax}$

Ns , * Não Significativo e significativo $\mathrm{p}<0,05$, respectivamente.

(1- 'Natal', 2- 'Valência', 3- 'Valência-Don João', 4- 'Valência-late-Burjasot' IVIA 35-2, 5- 'Valência-Rohde-Red' SRA-36, 6- 'Valência-Temprana’ IVIA-25, 7- 'Valência-Campbell’). 
TABELA 5 - Estimativas dos parâmetros das equações polinomiais ajustadas para exprimir a relação de `Brix, acidez e ratio com o número de dias acumulados após o início da coleta de frutos, e correspondentes coeficientes de determinação, para as seleções e variedades de laranjas, enxertadas sobre tangerineira Sunki. (Experimento II) Bebedouro-SP, 2006.

\begin{tabular}{|c|c|c|c|c|c|c|c|c|c|c|}
\hline \multirow{2}{*}{ Tratamento } & \multicolumn{4}{|c|}{${ }^{\circ}$ Brix $^{1}$} & \multicolumn{3}{|c|}{ Acidez $^{2}$} & \multicolumn{3}{|c|}{ Ratio $^{3}$} \\
\hline & c & $\mathrm{b}$ & a & $\mathrm{r}^{2}$ & $\mathrm{~b}$ & $\mathrm{a}$ & $\mathrm{r}^{2}$ & $\mathrm{~b}$ & $\mathrm{a}$ & $\mathrm{r}^{2}$ \\
\hline 1 & 10,2133 & 0,05874 & $-0,000342$ & $89,66^{*}$ & 1,5326 & $-0,00557$ & $98,18^{*}$ & 6,5277 & 0,0668 & $98,75^{*}$ \\
\hline 2 & 9,7844 & 0,0612 & - 0,000399 & $96,03 *$ & 1,364 & $-00,00475$ & $89,70^{*}$ & 7,2626 & 0,0632 & $97,03 *$ \\
\hline 3 & 10,2817 & 0,06738 & $-0,000437$ & $70,51^{*}$ & 1,9069 & $-0,006251$ & $80,86^{*}$ & 5,9074 & 0,0388 & $93,75^{*}$ \\
\hline 4 & 9,5484 & 0,0489 & $-0,000312$ & $95,58^{*}$ & 1,1931 & $-0,004181$ & $94,85^{*}$ & 7,9947 & 0,0686 & $98,09 *$ \\
\hline 5 & 10,451 & 0,04892 & $-0,000314$ & $69,74^{*}$ & 1,3758 & $-0,005396$ & $94,23 *$ & 6,8604 & 0,0778 & $97,13 *$ \\
\hline 6 & 9,5259 & 0,0832 & $-0,00044$ & $79,95^{*}$ & 1,244 & $-0,001886$ & $28,59 \mathrm{NS}$ & 8,8274 & 0,00345 & $77,50^{*}$ \\
\hline 7 & 10,2653 & 0,06 & $-0,000385$ & $90,92 *$ & 1,6566 & $-0,0060$ & $88,73^{*}$ & 6,2783 & 0,0619 & $95,90 *$ \\
\hline
\end{tabular}

$1{ }^{\circ}$ Brix $\mathrm{y}=\mathrm{c}+\mathrm{bx}+\mathrm{ax}^{2} ;{ }^{2}$ Acidez (\%) $\mathrm{y}=\mathrm{b}+\mathrm{ax} ;{ }^{3}$ Ratio $\mathrm{y}=\mathrm{b}+\mathrm{ax}$

Ns , * Não Significativo e significativo $\mathrm{p}<0,05$, respectivamente.

(1- 'Natal', 2- 'Valência', 3- 'Valência-Don João', 4- 'Valência-late-Burjasot' IVIA 35-2, 5-'Valência-Rohde-Red’ SRA-36, 6- 'Valência-Temprana’ IVIA-25, 7- 'Valência-Campbell’).

\section{CONCLUSÕES}

1-Nas condições do experimento, conclui-se que:

2-As seleções apresentaram potencial promissor, quanto às variáveis estudadas, para indústria e consumo in natura, com destaque para a seleção 'Valência late Burjasot’ IVIA 35-2, a que mais se assemelhou às variedades tradicionais Valência e Natal.

\section{REFERÊNCIAS}

ABECITRUS. Exportação de FCOJ-S: histórico. Disponível em: <http://www.abecitrus.com.br>. Acesso em: 26 abr. 2008

AGRIANUAL 2007: anuário da agricultura brasileira. São Paulo: FNP Consultoria \& Comércio, 2007. p.277297.

AGRIANUAL 2008: Anuário da Agricultura Brasileira. São Paulo: FNP Consultoria \& Comércio, 2008. p.273-303.

BOTEON, M.; NEVES, E. M. Citricultura brasileira: aspectos econômicos. In: JUNIOR, D. D. M.; DE NEGRI, J. D.; PIO, R. M.; JUNIOR, J. P. Citros: liderança paulista. Campinas: Instituto Agronômico e Fundag, 2005. p.20-36.

DI GIORGI, F.; IDE, B. Y.; DIB, K.; MARKI, R. J.; TRIBONI, H. R.; WAGNER, R. L. Contribuição ao estudo do comportamento de algumas variedades de citros e suas implicações. Laranja, Cordeirópolis, v.11.n.2, p.567-612, 1990.

DOMINGUES, E. T. Caracterização morfológica, agronômica, isoenzimática e por RAPD de variedades de laranja-doce - Citrus sinensis (L) Osbeck. Piracicaba, SP. 1998. 251 f. Tese (Doutor em genética e Melhoramento de Plantas) - Escola Superior de Agricultura “Luiz de Queiroz”, Universidade de São Paulo, Piracicaba, 1998.

JONES, W.W. Environmental and cultural factors unfluencing the chemical composition and physical characters. In: SINCLAIR, W.B. (Ed.). The orange: its biochemistry and physiology. Berkeley: University of California, 1961. p.25-55.

MATTOS JUNIOR, D.; GONZALES, A. F.; POMPEU JUNIOR, J.; PARAZZI, C. Avaliação de curvas de 
maturação de laranjas por análises de agrupamento. Pesquisa Agropecuária Brasileira, Brasília, v.34, n.12, p.2203-2209, 1999.

NEVES, E. M. Importância socioeconômica da citricultura no Estado de São Paulo. 1997. Disponível em: <http://www.watson.fapesp.br/Evaristo.htm>. Acesso em: 26 abr. 2008.

NEVES, E.M., RODRIGUES, K.; DRAGONE, D.S.; DAYOUB, M. Citricultura: geração de divisas e formação de renda no plano real. Laranja, Cordeirópolis. v.24, n.2, p.253-266, 2003.
SINCLAIR, W. B. Principal juice componets. In: SINCLAIR, W. B. (Ed.). The orange: its biochemistry and physiology. Berkeley: University of California, 1961.p.131-160.

STUCHI, E. S.; DONADIO, L. C.; SEMPIONATO, O. R. Qualidade industrial e produção de frutos de laranjeira 'Valência' enxertada sobre sete portasenxerto. Laranja, Cordeirópolis. b23, n.2, p.453-471, 2002.

VIÉGAS, F. C. P. Compra da laranja através dos sólidos solúveis. Laranja, Cordeirópolis, SP. v.2, n.9, p.467-481.1991. 